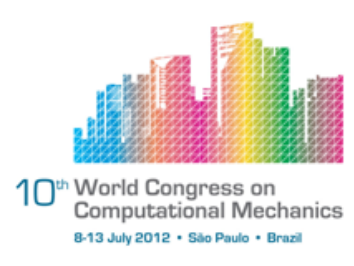

\title{
RETURN MAPPING FOR CREEP AND PLASTICITY SPLIT
}

\author{
V. D. Fachinotti ${ }^{1}$, A. E. Albanesi ${ }^{2}$, A. Cardona ${ }^{2}$ \\ ${ }^{1}$ International Center for Computational Methods in Engineering (CIMEC), Institute for the \\ Technological Development of the Chemical Industry (INTEC), dependent on National Lit- \\ toral University (UNL) and National Council for Scientific and Technical Research (CON- \\ ICET), Santa Fe, Argentina (vfachino@intec.unl.edu.ar)
}

${ }^{2}$ CIMEC-INTEC, UNL/CONICET, Santa Fe, Argentina

\begin{abstract}
Within the range of validity of the small strain theory, the strain is additively decomposed in an elastic or reversible strain and an inelastic or irreversible strain. The inelastic strain consists of a plastic component quickly developed under loading, and a viscous or creep component that develops slowly under loading. Most frequently, both components are assumed to develop simultaneously following a unique flow rule for the whole inelastic strain according to the Bingham-Norton rheological model, which neglects creep recovery. Under experimental evidence of considerable creep recovery, instantaneous plastic strain and slow creep evolve according their own flow rules. Unlike the case Bingham-Norton models are used, it is no longer possible to define a trial stress state from which it can be determined whether the plastic strain has increased or not under a given load increment. We introduce a new "non-plastic" trial state, which differs from the classical elastic trial state (Simo and Hughes, Computational Inelasticity, Springer-Verlag, 1998) since creep increment is now allowed. In order to explicit the "non-plastic" trial state, the creep flow rule, generally nonlinear, is solved. From the "non-plastic" trial state, two alternatives can be derived: (i) no plastic increment has been produced (in such a case, the trial state is actually the solution); (ii) there must be plastic increment, and therefore the stress cannot exceed the yield stress. In the last case, the creep flow rule is supplemented by the plastic consistency condition, giving rise to a system of non-linear scalar equations, to be solved for determining the actual plastic and creep increment under the given load.
\end{abstract}

Keywords: return mapping, creep, plasticity.

\section{INTRODUCTION}

Most of combined creep and plasticity models are based on the Bingham-Norton rheological model [1]. This model embodies creep and plasticity strain in the so-called viscoplastic strain, which constitutes the whole irreversible or inelastic part of the total strain. The 
Bingham-Norton models succeed to represent the behavior of most metals at high temperature, and it was widely used in the author's previous work [2, 3, 4, 5] for modeling steel casting processes.

However, the Bingham-Norton models neglect creep recovery, and hence they do not represent satisfactorily the behavior of certain materials, including those used in nuclear fuel rods we are currently interested in. Then, in such cases, a model that distinguishes creep and plasticity strains, each one having its own flow rule, must be used.

To this end, we develop a three-dimensional generalization of the one-dimensional flow rules proposed by Soba and Denis [6] to characterize the behavior of the materials used in fuel rods in nuclear power plants in Argentina. Finally, a specialized return-mapping algorithm to deal with separated creep and plasticity is proposed.

\section{CONSTITUTIVE MODELING}

Assuming the validity of the small strain theory, the total strain tensor $\varepsilon$ can be additively decomposed as

$$
\varepsilon=\varepsilon^{\mathrm{el}}+\varepsilon^{\mathrm{in}}
$$

where $\varepsilon^{\mathrm{el}}$ is the elastic (reversible) strain tensor and $\varepsilon^{\text {in }}$ is the inelastic (irreversible) strain tensor.

Moreover, the inelastic strain is decomposed in the plastic (instantaneous) strain $\varepsilon^{\mathrm{pl}}$ and the creep strain $\varepsilon^{\text {cr }}$, i.e.

$$
\varepsilon^{\mathrm{in}}=\varepsilon^{\mathrm{pl}}+\varepsilon^{\mathrm{cr}}
$$

Once the strain components are determined, the stress tensor $\sigma$ can be computed using the Hooke's law:

$$
\boldsymbol{\sigma}=2 \mu \operatorname{dev}\left(\varepsilon^{\mathrm{el}}-\varepsilon^{0}\right)+\kappa \operatorname{tr}\left(\varepsilon^{\mathrm{el}}-\varepsilon^{0}\right) \boldsymbol{I}
$$

where $\mu$ is the material shear modulus, $\kappa$ is the material bulk modulus, $\varepsilon^{0}$ is the initial strain tensor, $\boldsymbol{I}$ is the second-rank identity tensor, $\operatorname{dev}(*)$ and $\operatorname{tr}(*)$ denote the deviator and the trace of tensor $(*)$. Let us note that the first term of the r.h.s. of equation (3) defines the deviatoric stress $s=\operatorname{dev}(\boldsymbol{\sigma})$, while the second term defines the hydrostatic stress.

Regarding the initial strain $\varepsilon^{0}$, it is composed of the thermally-induced strain $\varepsilon^{\text {th }}$ as usual but also of contributions that are specific to the materials being modeled, like the swelling strain $\varepsilon^{\mathrm{sw}}$ and the densification strain $\varepsilon^{\mathrm{d}}$ in the the pellet and the irradiation-growth strain $\varepsilon^{\mathrm{ig}}$ in the cladding of nuclear fuel rods. Let us remark that $\varepsilon^{0}$ is not generally isotropic: while $\varepsilon^{\text {th }}$ is usually assumed isotropic, Soba and Denis [6] assume that $\varepsilon^{\mathrm{sw}}$ and $\varepsilon^{\mathrm{d}}$ are also isotropic but $\varepsilon^{\mathrm{ig}}$ is not.

\subsection{Evolution of plastic strain}

As aforementioned, the plastic and creep components of strain evolve according their own flow rules.

First, plastic strain is assumed to evolve whenever the Von-Mises yield criterion $f=0$ is met, where

$$
f=\sigma_{\mathrm{eq}}-\sigma_{\mathrm{Y}}-R
$$


where $\sigma_{\mathrm{eq}}=\sqrt{3 / 2}\|s\|$ is the Von-Mises equivalent stress, and $\sigma_{\mathrm{Y}}+R$ is the instantaneous yield stress, i.e., the initial yield stress $\sigma_{\mathrm{Y}}$ plus $R$ from isotropic hardening. We assume $R=$ $R\left(\varepsilon_{\mathrm{eq}}^{\mathrm{pl}}\right)$, where $\varepsilon_{\mathrm{eq}}^{\mathrm{pl}}$ is the plastic strain accumulated up to time $t$ :

$$
\varepsilon_{\mathrm{eq}}^{\mathrm{pl}}=\sqrt{2 / 3} \int_{0}^{t}\left\|\dot{\varepsilon}^{\mathrm{pl}}\right\| \mathrm{d} \tau
$$

Then, the evolution of plastic strain is assumed to obey the normality rule:

$$
\dot{\varepsilon}^{\mathrm{pl}}=\lambda \boldsymbol{n}
$$

where $\boldsymbol{n}=\boldsymbol{s} /\|\boldsymbol{s}\|$ and $\lambda$ is the plastic consistency parameter that satisfies the Kuhn-Tucker conditions:

$$
\lambda \geq 0, \quad f \leq 0, \quad \lambda \dot{f}=0
$$

\subsection{Evolution of creep}

In general, creep flow is not longer deviatoric as defined by the normality rule since it must account for the volume change induced by phenomena like densification in the pellet [7]. Therefore, a general flow rule for the materials used in nuclear fuel rods could be written as follows:

$$
\dot{\boldsymbol{\varepsilon}}^{\mathrm{cr}}=\sqrt{3 / 2} \dot{\varepsilon}_{\mathrm{eq}}^{\mathrm{cr}} \boldsymbol{n}+\frac{\dot{V}}{3} \boldsymbol{I}
$$

where $\dot{V}$ is the rate of volume change, and $\varepsilon_{\mathrm{eq}}^{\mathrm{cr}}$ is defined as

$$
\varepsilon_{\mathrm{eq}}^{\mathrm{cr}}=\sqrt{2 / 3} \int_{0}^{t}\left\|\operatorname{dev}\left(\dot{\varepsilon}^{\mathrm{cr}}\right)\right\| \mathrm{d} \tau
$$

From now on, $\dot{V}$ will be considered as a given parameter, which can be computed in terms of the evolution of porosity as proposed by Soba and Denis [6].

\subsubsection{Creep in nuclear fuel rod materials}

Let us consider the nuclear fuel rod studied by Soba and Denis [6], which is typically used in nuclear plants in Argentina, consisting of a $\mathrm{U}_{2} \mathrm{O}$ pellet inside a Zircaloy- 4 cladding.

The one-dimensional evolution of the creep strain $\varepsilon^{\mathrm{cr}}$ in $\mathrm{U}_{2} \mathrm{O}$ depends on the stress $\sigma$ and the (absolute) temperature $T$ according to the law:

$$
\dot{\varepsilon}^{\text {cr }}=\left[\frac{a_{1}+a_{2} \dot{f}}{\left(a_{3}+D\right) g^{2}} \exp \frac{-Q_{1}}{R T}+a_{7} \dot{f} \exp \frac{Q_{3}}{R T}\right] \sigma+\left(\frac{a_{1}+a_{8} \dot{f}}{a_{6}+D} \exp \frac{Q_{2}}{R T}\right) \sigma^{4.5}
$$

where $a_{i}$ is a material constant, $Q_{i}$ is an activation anergy, $R$ is the gas constant, $\dot{f}$ is the fission rate, $g$ is the grain size and $D$ is the percent of theoretical density.

On the other hand, the creep rate in Zircaloy-4 is assumed to depend not only on $\sigma$ and $T$ but also on the instantaneous creep. This is described by the one-dimensional law:

$$
\dot{\varepsilon}^{\mathrm{cr}}=\frac{1}{\varepsilon^{\mathrm{cr}}}\left\{2 k \phi \exp \frac{-Q_{4}}{R T}[\sigma+b \exp (c \sigma)]\right\}^{2}
$$


where $k, b, c$ are constant, $Q_{4}$ is an activation anergy and $\phi$ is the neutron flux.

Observing equations (10) and (11), the one-dimensional evolution law for creep in both materials can be expressed generically as

$$
\dot{\varepsilon}^{\mathrm{cr}}=g\left(\sigma, \varepsilon^{\mathrm{cr}}\right)
$$

being $g$ a generally non-linear function of $\sigma$ and $\varepsilon^{\text {cr }}$.

In order to generalize this law to three-dimensional applications, $\sigma$ and $\varepsilon^{\mathrm{cr}}$ are assimilated to $\sigma_{\mathrm{eq}}$ and $\varepsilon_{\mathrm{eq}}^{\mathrm{cr}}$, respectively.

\section{RETURN MAPPING FOR SEPARATED PLASTICITY AND CREEP}

Let us assume that we know $\varepsilon^{\mathrm{cr}}, \varepsilon_{\mathrm{eq}}^{\mathrm{cr}}, \varepsilon^{\mathrm{pl}}$ and $\varepsilon_{\mathrm{eq}}^{\mathrm{pl}}$ at time $t_{0}$. At time $t=t_{0}+\Delta t$, once the total strain $\varepsilon$ is known, their components are updated using the implicit Backward-Euler time stepping scheme:

$$
\begin{aligned}
& \boldsymbol{\varepsilon}^{\mathrm{pl}}=\boldsymbol{\varepsilon}_{0}^{\mathrm{pl}}+\lambda \boldsymbol{n} \Delta t \\
& \varepsilon_{\mathrm{eq}}^{\mathrm{pl}}=\varepsilon_{\mathrm{eq}_{0}}^{\mathrm{pl}}+\sqrt{2 / 3} \lambda \Delta t \\
& \boldsymbol{\varepsilon}^{\mathrm{cr}}=\boldsymbol{\varepsilon}_{0}^{\mathrm{cr}}+\sqrt{3 / 2} g\left(\sigma_{\mathrm{eq}}, \varepsilon_{\mathrm{eq}}^{\mathrm{cr}}\right) \boldsymbol{n} \Delta t+\frac{\dot{V}}{3} \boldsymbol{I} \Delta t \\
& \varepsilon_{\mathrm{eq}}^{\mathrm{cr}}=\varepsilon_{\mathrm{eq}_{0}}^{\mathrm{cr}}+g\left(\sigma_{\mathrm{eq}}, \varepsilon_{\mathrm{eq}}^{\mathrm{cr}}\right) \Delta t
\end{aligned}
$$

where $(*)_{0}$ denotes the variable $(*)$ evaluated at time $t_{0}$, while the lack of subindex implies that the variable is evaluated at current time $t$.

As aforementioned, $\dot{V}$ will be considered as a given parameter. Therefore, the stress state at time $t$ is completely determined by the deviatoric stress

$$
\boldsymbol{s}=2 \mu \operatorname{dev}\left(\varepsilon-\varepsilon^{\text {in }}-\varepsilon^{0}\right)
$$

Now, let us define the "non-plastic" trial state by assuming no increment of the plastic strain in the time step $\left[t_{0}, t\right]$, i.e., $\varepsilon^{\mathrm{pl}}=\varepsilon_{0}^{\mathrm{pl}}$ and $\varepsilon_{\mathrm{eq}}^{\mathrm{pl}}=\varepsilon_{\mathrm{eq}_{0}}^{\mathrm{pl}}$. Let $\varepsilon^{\mathrm{cr}, \mathrm{np}}$ and $\varepsilon_{\mathrm{eq}}^{\mathrm{crnp}}$ be the corresponding values of $\varepsilon^{\mathrm{cr}}$ and $\varepsilon_{\mathrm{eq}}^{\mathrm{cr}}$. The "non-plastic" trial state is then determined by the deviatoric stress

$$
\boldsymbol{s}^{\mathrm{np}}=2 \mu \operatorname{dev}\left(\varepsilon-\varepsilon_{0}^{\mathrm{pl}}-\varepsilon^{\mathrm{cr}, \mathrm{np}}-\varepsilon^{0}\right)=\boldsymbol{s}^{\mathrm{el}}-\sqrt{6} \mu g\left(\sigma_{\mathrm{eq}}^{\mathrm{np}}, \varepsilon_{\mathrm{eq}}^{\mathrm{crnp}}\right) \boldsymbol{n} \Delta t
$$

where $\sigma_{\mathrm{eq}}^{\mathrm{np}}=\sqrt{3 / 2}\left\|s^{\mathrm{np}}\right\|$ (making above equation non-linear for $s^{\mathrm{np}}$ ), and

$$
\boldsymbol{s}^{\mathrm{el}}=2 \mu \operatorname{dev}\left(\varepsilon-\varepsilon_{0}^{\mathrm{pl}}-\varepsilon_{0}^{\mathrm{cr}}-\varepsilon^{0}\right)
$$

defines the "elastic" trial state, that obtained assuming no inelastic (plastic and creep) increment during $\left[t_{0}, t\right]$, which coincides with the classical definition of Simo and Hughes [8] for unified creep and plasticity. Since all the strains involved in equation (19) are known at this stage, $s^{\text {el }}$ can be easily computed.

Let us remark that $s, s^{\mathrm{np}}$ and $s^{\mathrm{el}}$ are co-linear, i.e.:

$$
\frac{s}{\|s\|}=\frac{s^{\mathrm{np}}}{\left\|s^{\mathrm{np}}\right\|}=\frac{s^{\mathrm{el}}}{\| s^{\mathrm{el} \|}}=n
$$


Using this property, the tensor equation (18) reduces to the scalar equation

$$
\sigma_{\mathrm{eq}}^{\mathrm{np}}=\sigma_{\mathrm{eq}}^{\mathrm{el}}-3 \mu\left(\varepsilon_{\mathrm{eq}}^{\mathrm{cr}, \mathrm{np}}-\varepsilon_{\mathrm{eq}_{0}}^{\mathrm{cr}}\right) \equiv \sigma_{\mathrm{eq}}^{\mathrm{np}}\left(\varepsilon_{\mathrm{eq}}^{\mathrm{cr}, \mathrm{np}}\right)
$$

with $\sigma_{\text {eq }}^{\text {el }}=\sqrt{3 / 2}\left\|s^{\mathrm{el}}\right\|$ (already known).

If we replace $\sigma_{\mathrm{eq}}^{\mathrm{np}}$ as defined by equation (21) into the discrete creep flow rule given by equation (16), the following non-linear scalar equation with $\varepsilon_{\mathrm{eq}}^{\mathrm{cr}, \mathrm{pp}}$ as unique unknown is obtained:

$$
F_{1}\left(\varepsilon_{\mathrm{eq}}^{\mathrm{cr}, \mathrm{np}}\right)=\varepsilon_{\mathrm{eq}}^{\mathrm{cr}, \mathrm{pp}}-\varepsilon_{\mathrm{eq}_{0}}^{\mathrm{cr}}-g\left(\sigma_{\mathrm{eq}}^{\mathrm{np}}\left(\varepsilon_{\mathrm{eq}}^{\mathrm{crnp}}\right), \varepsilon_{\mathrm{eq}}^{\mathrm{crnp}}\right) \Delta t=0
$$

In general, this equation has not a closed analytical solution. We solve it using the secant or regula-falsi method [9], which has proved to be efficient.

Once $\varepsilon_{\mathrm{eq}}^{\mathrm{cr} n \mathrm{np}}$ is determined, and so is $\sigma_{\mathrm{eq}}^{\mathrm{np}}$, we evaluate the yield function for the "nonplastic" trial state:

$$
f^{\mathrm{np}} \equiv f\left(\sigma_{\mathrm{eq}}^{\mathrm{np}}, \varepsilon_{\mathrm{eq}_{0}}^{\mathrm{pl}}\right)=\sigma_{\mathrm{eq}}^{\mathrm{np}}-\sigma_{\mathrm{Y}}-R\left(\varepsilon_{\mathrm{eq}_{0}}^{\mathrm{pl}}\right)
$$

If we assume $R$ to be a non-decreasing function of $\varepsilon_{\mathrm{eq}}^{\mathrm{pl}}$ (i.e., material softening is not allowed), it can be easily derived that

$$
f \leq f^{\mathrm{np}}
$$

Then, regarding the sign of $f^{\text {np }}$, two different situations can be inferred:

1. If $f^{\text {np }}<0$, then $f<0$ and hence no plastic increment takes place during the step $\left[t_{0}, t\right]$. In such a case, the "non-plastic" trial state is actually the real state.

2. If $f^{\text {np }} \geq 0$, then $f=0$ and

$$
\sigma_{\mathrm{eq}}=\sigma_{\mathrm{Y}}+R\left(\varepsilon_{\mathrm{eq}}^{\mathrm{pl}}\right)
$$

Now, the equation (17) defining the stress deviator can be rewritten as follows:

$$
\boldsymbol{s}=\boldsymbol{s}^{\mathrm{el}}-2 \mu \lambda \boldsymbol{n} \Delta t-\sqrt{6} g\left(\sigma_{\mathrm{eq}}^{\mathrm{np}}, \varepsilon_{\mathrm{eq}}^{\mathrm{cr}, \mathrm{np}}\right) \boldsymbol{n} \Delta t
$$

Since $s, s^{\mathrm{el}}$ and $\boldsymbol{n}$ are co-linear, the above tensor equation reduces to the scalar equation:

$$
\sigma_{\mathrm{eq}}=\sigma_{\mathrm{eq}}^{\mathrm{el}}-3 \mu\left(\varepsilon_{\mathrm{eq}}^{\mathrm{pl}}-\varepsilon_{\mathrm{eq}_{0}}^{\mathrm{pl}}\right)-3 \mu\left(\varepsilon_{\mathrm{eq}}^{\mathrm{cr}}-\varepsilon_{\mathrm{eq}_{0}}^{\mathrm{cr}}\right)
$$

Equating equations (25) and (27), we obtain

$$
\varepsilon_{\mathrm{eq}}^{\mathrm{cr}}=\frac{1}{3 \mu}\left[\sigma_{\mathrm{eq}}^{\mathrm{el}}-\sigma_{\mathrm{Y}}-R\left(\varepsilon_{\mathrm{eq}}^{\mathrm{pl}}\right)\right]-\varepsilon_{\mathrm{eq}}^{\mathrm{pl}}+\varepsilon_{\mathrm{eq}_{0}}^{\mathrm{cr}}+\varepsilon_{\mathrm{eq}_{0}}^{\mathrm{pl}} \equiv \varepsilon_{\mathrm{eq}}^{\mathrm{cr}}\left(\varepsilon_{\mathrm{eq}}^{\mathrm{pl}}\right)
$$

If we replace this relationship between $\varepsilon_{\mathrm{eq}}^{\mathrm{cr}}$ and $\varepsilon_{\mathrm{eq}}^{\mathrm{pl}}$ into the discrete creep flow rule given by equation (16), the following non-linear scalar equation with $\varepsilon_{\mathrm{eq}}^{\mathrm{pl}}$ as unique unknown is obtained:

$$
F_{2}\left(\varepsilon_{\mathrm{eq}}^{\mathrm{pl}}\right)=\varepsilon_{\mathrm{eq}}^{\mathrm{cr}}\left(\varepsilon_{\mathrm{eq}}^{\mathrm{pl}}\right)-\varepsilon_{\mathrm{eq}_{0}}^{\mathrm{cr}}-g\left(\sigma_{\mathrm{Y}}+R\left(\varepsilon_{\mathrm{eq}}^{\mathrm{pl}}\right), \varepsilon_{\mathrm{eq}}^{\mathrm{cr}}\left(\varepsilon_{\mathrm{eq}}^{\mathrm{pl}}\right)\right) \Delta t=0
$$

As before, this equation can be solved using a secant method. 


\subsection{Consistent tangent moduli}

The tangent moduli is defined as

$$
\frac{\partial \sigma_{i j}}{\partial \varepsilon_{k l}}=\frac{\partial s_{i j}}{\partial \varepsilon_{k l}}+\kappa \delta_{i j} \delta_{k l}
$$

Contributions from $\dot{V}$, if any, are not considered here. Then, it remains to compute

$$
\frac{\partial s_{i j}}{\partial \varepsilon_{k l}}=\|\boldsymbol{s}\| \frac{\partial n_{i j}}{\partial \varepsilon_{k l}}+\frac{\partial\|s\|}{\partial \varepsilon_{k l}} n_{i j}
$$

Considering $n=s^{\mathrm{el}} /\left\|s^{\mathrm{el}}\right\|$, we have

$$
\frac{\partial n_{i j}}{\partial \varepsilon_{k l}}=\frac{1}{\left\|s^{\mathrm{el}}\right\|} \frac{\partial s_{i j}^{\mathrm{el}}}{\partial \varepsilon_{k l}}-\frac{n_{i j}}{\left\|\boldsymbol{s}^{\mathrm{e} l}\right\|} \frac{\partial\left\|\boldsymbol{s}^{\mathrm{el}}\right\|}{\partial \varepsilon_{k l}}
$$

With $s^{\text {el }}$ defined by equation (19), its derivative is

$$
\frac{\partial s_{i j}^{\mathrm{el}}}{\partial \varepsilon_{k l}}=2 \mu\left[\frac{1}{2}\left(\delta_{i k} \delta_{j l}+\delta_{i l} \delta_{j k}\right)-\frac{1}{3} \delta_{i j} \delta_{k l}\right] \equiv 2 \mu I_{i j k l}^{\mathrm{dev}}
$$

and then

$$
\frac{\partial\left\|s^{\mathrm{el}}\right\|}{\partial \varepsilon_{k l}}=n_{i j} \frac{\partial s_{i j}^{\mathrm{el}}}{\partial \varepsilon_{k l}}=2 \mu n_{k l}
$$

Finally, equation (32) becomes explicitely

$$
\frac{\partial n_{i j}}{\partial \varepsilon_{k l}}=\frac{2 \mu}{\| \boldsymbol{s}^{\mathrm{e} \mathrm{l} \|}}\left(I_{i j k l}^{\mathrm{dev}}-n_{i j} n_{k l}\right)
$$

On the other hand, when differentiating $\|s\|$, distinction must be made based on the yield criterion, as detailed below.

1. For $f<0$, we have $\|s\| \equiv\left\|s^{\mathrm{np}}\right\|$ and then, taking derivatives in equation (21), we have

$$
\frac{\partial\|s\|}{\partial \varepsilon_{k l}}=\frac{\partial\left\|s^{\mathrm{el}}\right\|}{\partial \varepsilon_{k l}}-\sqrt{6} \mu \frac{\partial \varepsilon_{\mathrm{eq}}^{\mathrm{cr}}}{\partial \varepsilon_{k l}}
$$

Invoking the discrete creep flow rule, equation (16), we have

$$
\frac{\partial \varepsilon_{\mathrm{eq}}^{\mathrm{cr}}}{\partial \varepsilon_{k l}}=\sqrt{3 / 2} \frac{\partial g}{\partial \sigma_{\mathrm{eq}}} \frac{\partial\|s\|}{\partial \varepsilon_{k l}} \Delta t+\frac{\partial g}{\partial \varepsilon_{\mathrm{eq}}^{\mathrm{cr}}} \frac{\partial \varepsilon_{\mathrm{eq}}^{\mathrm{cr}}}{\partial \varepsilon_{k l}} \Delta t
$$

By replacing equation (36) into equation (37), we obtain

$$
\frac{\partial \varepsilon_{\mathrm{eq}}^{\mathrm{cr}}}{\partial \varepsilon_{k l}}=\frac{1-\Theta^{\mathrm{cr}}}{\sqrt{6} \mu} \frac{\partial\left\|s^{\mathrm{el}}\right\|}{\partial \varepsilon_{k l}}=\sqrt{2 / 3}\left(1-\Theta^{\mathrm{cr}}\right) n_{k l}
$$

with

$$
\Theta^{\mathrm{cr}}=1-\frac{\partial g}{\partial \sigma_{\mathrm{eq}}}\left(\frac{\partial g}{\partial \sigma_{\mathrm{eq}}}-\frac{1}{3 \mu} \frac{\partial g}{\partial \varepsilon_{\mathrm{eq}}^{\mathrm{cr}}}+\frac{1}{3 \mu \Delta t}\right)^{-1}
$$

Finally, the derivative of $\|s\|$ can be computed as

$$
\frac{\partial\|\boldsymbol{s}\|}{\partial \varepsilon_{k l}}=2 \mu \Theta^{\mathrm{cr}} n_{k l}
$$


2. If $f=0$, then $\sigma_{\mathrm{eq}} \equiv \sigma_{\mathrm{Y}}+R$, so that

$$
\frac{\partial\|s\|}{\partial \varepsilon_{k l}}=\sqrt{2 / 3} R^{\prime} \frac{\partial \varepsilon_{\mathrm{eq}}^{\mathrm{pl}}}{\partial \varepsilon_{k l}}
$$

where $R^{\prime}=\mathrm{d} R / \mathrm{d} \varepsilon_{\text {eq }}^{\mathrm{pl}}$.

By differentiating the discrete creep flow rule given as a function of $\varepsilon_{\mathrm{eq}}^{\mathrm{pl}}$ by given equation (28), we obtain

$$
\frac{\partial \varepsilon_{\mathrm{eq}}^{\mathrm{cr}}}{\partial \varepsilon_{k l}}=\frac{1}{\sqrt{6} \mu} \frac{\partial\left\|s^{\mathrm{el}}\right\|}{\partial \varepsilon_{k l}}-\left(1+\frac{R^{\prime}}{3 \mu}\right) \frac{\partial \varepsilon_{\mathrm{eq}}^{\mathrm{pl}}}{\partial \varepsilon_{k l}}
$$

Alternatively, this derivative can be computed by differentiating the discrete creep flow rule as given by equation (16):

$$
\frac{\partial \varepsilon_{\mathrm{eq}}^{\mathrm{cr}}}{\partial \varepsilon_{k l}}=\frac{\partial g}{\partial \varepsilon_{\mathrm{eq}}^{\mathrm{cr}}} \frac{\partial \varepsilon_{\mathrm{eq}}^{\mathrm{cr}}}{\partial \varepsilon_{k l}} \Delta t+\frac{\partial g}{\partial \sigma_{\mathrm{eq}}} R^{\prime} \frac{\partial \varepsilon_{\mathrm{eq}}^{\mathrm{pl}}}{\partial \varepsilon_{k l}}
$$

After equating equations (42) and (43), we obtain

$$
\frac{\partial \varepsilon_{\mathrm{eq}}^{\mathrm{pl}}}{\partial \varepsilon_{k l}}=\sqrt{3 / 2} \frac{1}{R^{\prime}} \Theta^{\mathrm{pl}+\mathrm{cr}} \frac{\partial\left\|s^{\mathrm{el}}\right\|}{\partial \varepsilon_{k l}}=\sqrt{6} \frac{\mu}{R^{\prime}} \Theta^{\mathrm{pl}+\mathrm{cr}} n_{k l}
$$

with

$$
\Theta^{\mathrm{pl}+\mathrm{cr}}=\frac{R^{\prime}}{3 \mu}\left[1+\frac{R^{\prime}}{3 \mu}+\frac{\partial g}{\partial \sigma_{\mathrm{eq}}} R^{\prime}\left(\frac{1}{\Delta t}-\frac{\partial g}{\partial \varepsilon_{\mathrm{eq}}^{\mathrm{cr}}}\right)^{-1}\right]^{-1}
$$

Finally, the derivative of $\|s\|$ can be computed as

$$
\frac{\partial\|s\|}{\partial \varepsilon_{k l}}=2 \mu \Theta^{\mathrm{pl}+\mathrm{cr}} n_{k l}
$$

\subsubsection{General expression of the tangent moduli}

Introducing the derivative of $\boldsymbol{n}$ given by equation (35) together with the derivative of $\|s\|$ given either by equation (40) for $f<0$ or equation (46) for $f=0$ into equation (31), and this one into equation (47), the tangent moduli can be expressed as

$$
\frac{\partial \sigma_{i j}}{\partial \varepsilon_{k l}}=2 \mu \frac{\|\boldsymbol{s}\|}{\| \boldsymbol{s}^{\mathrm{el} \|}} I_{i j k l}^{\mathrm{dev}}-2 \mu \Theta n_{i j} n_{k l}+\kappa \delta_{i j} \delta_{k l}
$$

where

$$
\Theta=\left\{\begin{array}{lll}
\Theta^{\text {cr }} & \text { (equation (39)) } & \text { if } f<0 \\
\Theta^{\text {pl+cr }} & \text { (equation (45)) } & \text { if } f=0
\end{array}\right.
$$


Table 1. Mechanical parameters of Zircaloy-4.

\begin{tabular}{ll}
\hline Material constants & $k=5.12 \times 10^{-29}$ \\
& $b=725.2$ \\
& $c=4.967 \times 10^{-8}$ \\
\hline Activation energy & $Q_{4}=10000 \mathrm{~J} / \mathrm{mol}$ \\
\hline Neutron flux & $\phi=5 \times 10^{15}$ neutrons $/\left(\mathrm{m}^{2} \mathrm{~s}\right)$ \\
\hline Shear modulus & $\mu=8.840 \times 10^{4}\left(1-1.0915 \times 10^{-4} T\right)$ \\
& $(\mu$ in $\mathrm{MPa}, T$ in $\mathrm{K})$ \\
\hline Bulk modulus & $\kappa=2.161 \times 10^{5}\left(1-1.0915 \times 10^{-4} T\right)$ \\
& $(\kappa$ in $\mathrm{MPa}, T$ in $\mathrm{K})$ \\
\hline Initial yield stress & $\sigma_{\mathrm{Y}}=200 \mathrm{MPa}$ \\
\hline
\end{tabular}

\section{APPLICATION}

Let us consider a bar of Zircaloy-4 with length $L=1 \mathrm{~m}$, which suffers a total extension $d=0.05 L$ that is applied using different extension rates. Any other source of deformation is neglected, i.e. $\varepsilon^{0}=0$ and $\dot{V}=0$.

Material properties, listed in Table 1, are those used by FRAPCON-3 [7], a widely used code for the thermo-mechanical analysis of nuclear fuel rods.

Four decreasing strain rates are considered, such that the extension of the bar is fully developed in one hour $\left(\dot{\varepsilon}=2.78 \times 10^{-4} / \mathrm{s}\right)$, one day $\left(\dot{\varepsilon}=1.16 \times 10^{-5} / \mathrm{s}\right)$, one week $(\dot{\varepsilon}=$ $\left.1.65 \times 10^{-6} / \mathrm{s}\right)$ or one month $\left(\dot{\varepsilon}=3.86 \times 10^{-7} / \mathrm{s}\right)$. These strain rates are representative of the whole range of strain rates expected along the life cycle of a nuclear fuel rod.

Figure 1 on the left depicts the creep vs. total strain at ambient temperature $(300 \mathrm{~K})$, where it is apparent that the maximum creep -corresponding to the minimum strain rate- is one-order smaller than the total strain. As a consequence, as shown on the right of Figure 1, the influence of creep on the stress at ambient temperature will be negligible all along the life cycle of Zircaloy-4 when it is used to make the cladding of a nuclear fuel rod .
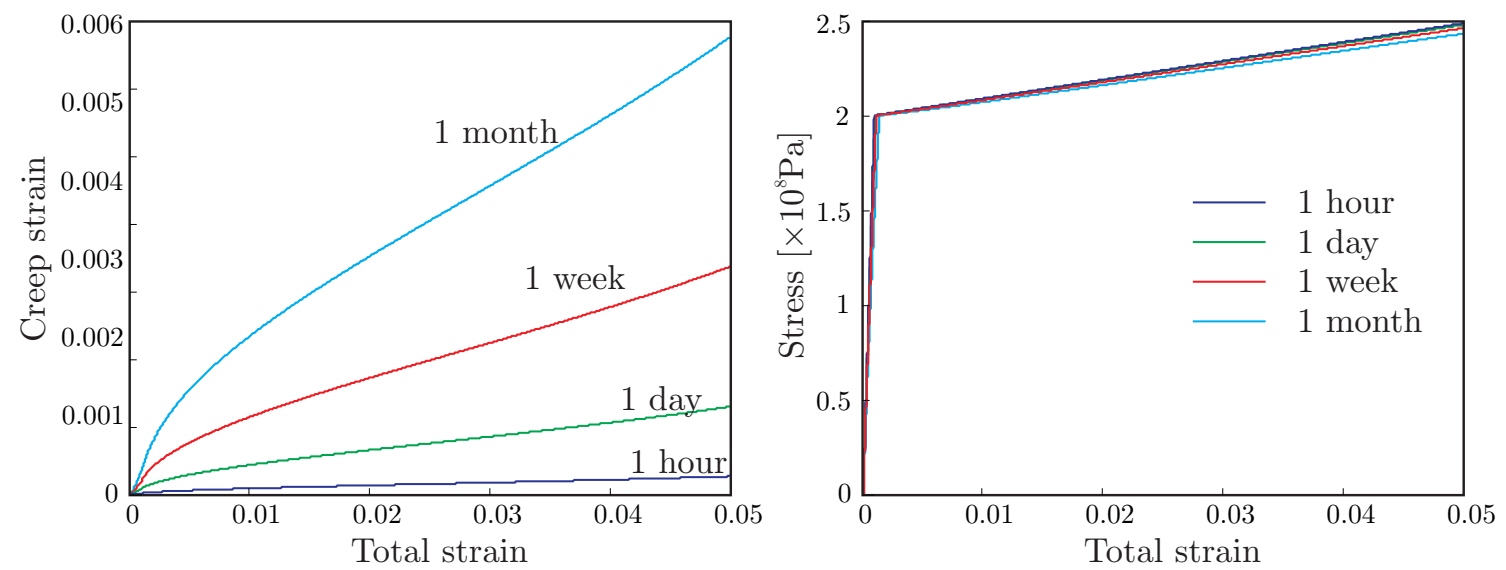

Figure 1. Creep strain and stress vs. total strain in an extended bar of Zircaloy-4 at ambient temperature $(300 \mathrm{~K})$, for different extension rates.

On the contrary, at high temperature $(1000 \mathrm{~K})$, creep strain constitutes an important fraction of the total strain, increasing for decreasing strain rates up to about $100 \%$ for the 
smaller rate (1/month), as shown on the left of Figure 2, with important consequences on the stress, as shown on the right of Figure 2.
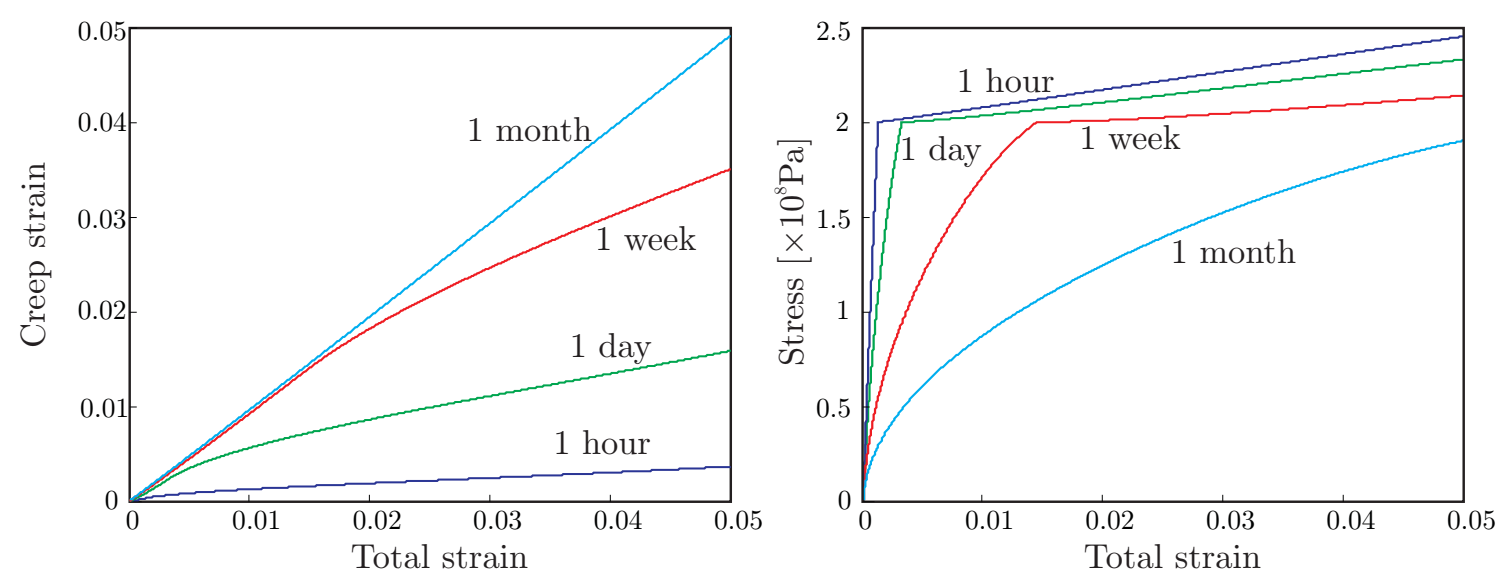

Figure 2. Creep strain and stress vs. total strain in an extended bar of Zircaloy-4 at high temperature $(1000 \mathrm{~K})$, for different extension rates.

\section{CONCLUSIONS}

We developed a return mapping algorithm addressed to integrate the evolution of creep and plasticity when they evolve separately. The algorithm is especially devoted to represent the behavior of materials used in nuclear fuel rods.

An application to Zircaloy-4 - used for the cladding of the rods- is discussed, with the aim of determining the influence of creep on the material behavior under temperatures and strain rates that are considered representative of those encountered during the life cycle of a nuclear fuel rod.

The model predicts -in agreement with experimental evidence- that creep has a negligible influence at ambient temperature, independently of the strain rate. The effect of creep increases as the temperature increases and the strain rate decreases. At a temperature of $1000 \mathrm{~K}$, a considerable influence of creep is predicted after a relatively short period of time.

\section{Acknowledgements}

This research was supported by the Nuclear Regulatory Agency (ARN) of Argentina.

\section{References}

[1] J. Lemaître and J.-L. Chaboche. Mechanics of Solid Materials. Cambridge University Press, 1994.

[2] A. E. Huespe, A. Cardona, N. Nigro, and V. D. Fachinotti. Visco-plastic constitutive models of steel at high temperature. J. Mat. Processing Tech., 102:143-152, 2000.

[3] V. D. Fachinotti and A. Cardona. Constitutive models of steel under continuous casting conditions. J. Mat. Processing Tech., 135:30-43, 2003. 
[4] M. Bellet and V. D. Fachinotti. ALE method for solidification modelling. Comput. Methods Appl. Mech. Engrg., 193:4355-4381, 2004.

[5] V. D. Fachinotti and A. Cardona. A fixed-mesh eulerian.lagrangian approach for stress analysis in continuous casting. Int. J. Numer. Meth. Engng., 70:728-755, 2007.

[6] A. Soba and A. Denis. Simulation with DIONISIO 1.0 of thermal and mechanical pelletcladding interaction in nuclear fuel rods. Journal of Nuclear Materials, 374:32-43, 2008.

[7] K. J. Geelhood, W. G. Luscher, and C. E. Beyer. FRAPCON-3.4: A Computer Code for the Calculation of Steady-State Thermal-Mechanical Behavior of Oxide Fuel Rods for High Burnup. United States Nuclear Regulatory Commission, 2011.

[8] J. C. Simo and T. J. R. Hughes. Computational Inelasticity. Springer-Verlag, 1998.

[9] O. C. Zienkiewicz and R. L. Taylor. The finite element method. Volume 2: Solid Mechanics. Butterworth-Heinemann, 5th edition, 2000. 